ARTICLE

\title{
Polymerized small molecular acceptor based all- polymer solar cells with an efficiency of $16.16 \%$ via tuning polymer blend morphology by molecular design
}

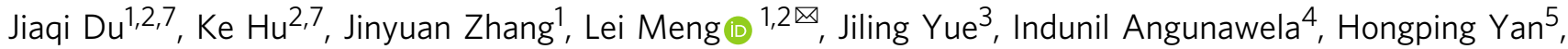
Shucheng Qin ${ }^{1,2}$, Xiaolei Kong ${ }^{1,2}$, Zhanjun Zhang ${ }^{2}$, Bo Guan ${ }^{3 凶}$, Harald Ade (i) ${ }^{4 凶}$ \& Yongfang Li (i) ${ }^{1,2,6 凶}$

\begin{abstract}
All-polymer solar cells (all-PSCs) based on polymerized small molecular acceptors (PSMAs) have made significant progress recently. Here, we synthesize two A-DA'D-A small molecule acceptor based PSMAs of PS-Se with benzo[c][1,2,5]thiadiazole $A^{\prime}$-core and PN-Se with benzotriazole $A^{\prime}$-core, for the studies of the effect of molecular structure on the photovoltaic performance of the PSMAs. The two PSMAs possess broad absorption with PN-Se showing more red-shifted absorption than PS-Se and suitable electronic energy levels for the application as polymer acceptors in the all-PSCs with PBDB-T as polymer donor. Cryogenic transmission electron microscopy visualizes the aggregation behavior of the PBDB-T donor and the PSMA in their solutions. In addition, a bicontinuous-interpenetrating network in the PBDB-T:PN-Se blend film with aggregation size of $10 \sim 20 \mathrm{~nm}$ is clearly observed by the photoinduced force microscopy. The desirable morphology of the PBDB-T:PN-Se active layer leads its all-PSC showing higher power conversion efficiency of $16.16 \%$.
\end{abstract}

\footnotetext{
${ }^{1}$ Beijing National Laboratory for Molecular Sciences, CAS Key Laboratory of Organic Solids, Institute of Chemistry, Chinese Academy of Sciences, Beijing, China. ${ }^{2}$ School of Chemical Science, University of Chinese Academy of Sciences, Beijing, China. ${ }^{3}$ Center for Physiochemical Analysis and Measurement, Institute of Chemistry, Chinese Academy of Sciences, Beijing, China. ${ }^{4}$ Department of Physics and Organic and Carbon Electronics Lab (ORaCEL), North Carolina State University, Raleigh, NC, USA. ${ }^{5}$ Department of Chemical Engineering, Stanford University, Stanford, CA, USA. ${ }^{6}$ Laboratory of Advanced Optoelectronic Materials, Suzhou Key Laboratory of Novel Semiconductor-Optoelectronics Materials and Devices, College of Chemistry, College of Chemistry, Chemical Engineering and Materials Science, Soochow University, Suzhou, Jiangsu, China. ${ }^{7}$ These authors contributed equally: Jiaqi Du, Ke Hu.

凶email: menglei@iccas.ac.cn; guanbo@iccas.ac.cn; hwade@ncsu.edu; liyf@iccas.ac.cn
} 
A ll-polymer solar cells (all-PSCs), based on the bulk heterojunction $(\mathrm{BHJ})$ active layers composed of a $p$-type conjugated polymer donor and an $n$-type conjugated polymer acceptor, have attracted growing attentions due to their advantages of good mechanical properties, photostability, and thermal stability compared with small molecule acceptor (SMA)based organic solar cells ${ }^{1-5}$. Recently, benefitted from the concept of polymerized small molecule acceptors (PSMAs) ${ }^{6,7}$, the power conversion efficiency (PCE) of the all-PSCs based on PSMA increases rapidly ${ }^{8-10}$, which has reached the threshold for commercialization and some specific applications like wearable and flexible devices ${ }^{11,12}$.

To establish high-performance all-PSCs, highly efficient polymer acceptor materials and the proper morphology of photoactive films are indispensable. In the development of polymer acceptors, an important breakthrough is the design strategy of PSMAs proposed by our group in $2017^{6}$, which is composed of a narrow bandgap SMA as main building block polymerized with a $\pi$-bridge linking unit. The PSMAs preserve the advantages of SMAs (such as narrow bandgap, strong absorption coefficient, and suitable electronic energy levels) and the advantages of polymers (such as higher stability and better flexibility) simultaneously ${ }^{7}$. The first PSMA PZ1 based on polymerized IDIC-C16 realized a PCE of $9.19 \%$ for the all-PSCs in $2017^{6}$, which was the highest PCE of the all-PSCs at that time. Then, by using PM6 as the polymer donor, the PCE of the PZ1-based allPSCs reached $11.2 \%^{13}$. After the A-DA'D-A structured SMA Y6 was reported in $2019^{14,15}$, the PSMAs based on the A-DA'D-A type SMA units have rapidly boosted the PCE of the all-PSCs up to over $15 \%^{16-18}$, owing to their narrower bandgap and smaller voltage loss. Thus, the selection of the small molecule acceptor building blocks plays a significant role in the photovoltaic properties of the PSMAs.

Moreover, a well-formed morphology of the all-polymer active layer with bicontinuous-interpenetrating network of polymer donor/polymer acceptor is essential to efficient exciton dissociation and charge carrier transport. In the PSCs based on small molecule acceptors, the strategies widely adopted for adjusting morphology involve addition of solvent additive, treatments of solvent annealing and thermal annealing, and processing with hot spin coating, etc ${ }^{19-21}$. However, compared with the blend films based on polymer donors and SMAs, most of the abovementioned methods for morphological control are not particularly effective in the all-PSCs, because both the polymer donor and polymer acceptor possess long molecular skeleton and stronger intermolecular interaction ${ }^{22}$, which often leads to preaggregation of the polymers in precursor blend solutions and becomes less sensitive to post-treatments of the polymer films. For example, the morphologies of the PSMAs of $\mathrm{PJ}^{23}$ and PTPBT-ET $_{\mathrm{x}}{ }^{24}$ exhibit insensitivity to the thermal annealing temperature or the volume ratio of solvent additives treatment in a certain range. Since intensive researches have revealed the interplay between the molecular structures and the blend film morphologies of the polymer donor and SMAs ${ }^{25,26}$, therefore the proper selection of SMA building blocks is critical in the design of PSMAs for modulating its electronic properties and adjusting its aggregation morphology.

As discussed above, PSMAs with suitable aggregation and good solubility should be developed for realizing high-performance allPSCs. So far, however, most of the latest reported PSMAs were based on the A-DA'D-A-structured Y6-like building block containing benzo[c] $[1,2,5]$ thiadiazole (BS) $\mathrm{A}^{\prime}$-core in their DA'D fused ring. Earlier studies have illustrated that the Y6 molecule has strong intermolecular packing ${ }^{27}$, and may cause difficulty in the balance of aggregation and solubility for the PSMAs. To address this issue, we consider to switch the building block to
Y18-like unit containing benzotriazole (BN) A'-core ${ }^{28}$ with an additional alkyl side chain on the top nitrogen of $\mathrm{BN}$ core, and Y18 is developed by our group in collaboration with Zou's group recently ${ }^{28}$. The one more alkyl side chain on the Y18-like unit (totally five side chains) could provide more possibilities of adjusting the aggregation and solubility, and could facilitate the modulation of the film morphology. Moreover, Y18 shows a slightly narrower bandgap than Y6, while the PM6:Y18 based PSCs display a higher open-circuit voltage $\left(V_{\mathrm{oc}}\right)$ than that of the PM6:Y6 based devices benefitted from its lower energy loss ${ }^{28}$. The advantages of Y18 on the tunable aggregation, broad absorption and lower energy loss could be brought into its corresponding PSMAs $^{18}$.

Based on above considerations, in this work, we designed and synthesized a new PSMA PN-Se (see Fig. 1a) with the A-DA'D-A type Y18-like unit (with BN A'-core) as main building block and selenophene as $\pi$-linking units in considering the red-shifted absorption of selenophene ${ }^{29}$. And we synthesized an analogue PSMA PS-Se (see Fig. 1a) with the Y6-like unit (with BS A'-core) as the main building block, for the studies of the A'-core structure on the photovoltaic performance of the PSMAs. As mentioned above, the $\mathrm{BN}$ unit in $\mathrm{PN}-\mathrm{Se}$ has one more alkyl side chain compared with the BS unit in PS-Se, which could affect the molecular packing of the corresponding polymer acceptors and the morphology of the blend film of the polymer acceptors with polymer donor. We compared the aggregation behavior of the two PSMAs by variable-temperature UV-vis absorption spectroscopy and by Cryogenic transmission electron microscopy (CryoTEM) in the solutions. In the all-PSCs, the preaggregated microstructure of the polymer donor and polymer acceptor in their blend precursor solution is generally considered to dominate the active layer film morphology $y^{30-32}$. However, there is no report on direct observation or study of these preaggregation state of the polymers blend in their solutions till now. Cryo-TEM is a technique to visualize the aggregation behavior of the molecules in solution by measuring the TEM morphology of the frozen solution sample 33,34 . Here, for the first time, we measured the morphology of the polymer micro-aggregates in the rapidly frozen blend precursor solutions of polymer donor and PSMAs by the Cryo-TEM. In addition, photo-induced force microscopy $(\mathrm{PiFM})^{35}$ was employed to characterize the morphology of the polymer blend films and to distinguish the phase region of the donor and acceptor polymers. Based on these measurements, we found that the PSMA PN-Se polymer acceptor exhibited more suitable phase separation and red-shifted absorption. The all-PSC based on PN-Se as polymer acceptor and PBDB- $\mathrm{T}^{36}$ as polymer donor demonstrated a high PCE of $16.16 \%$ with a $V_{o c}$ of $0.907 \mathrm{~V}$, $J_{s c}$ of $24.82 \mathrm{~mA} \mathrm{~cm}^{-2}$, and FF of 0.718 , while the PS-Se-based allPSC showed a moderate PCE of $13.83 \%$ with a $V_{o c}$ of $0.874 \mathrm{~V}, J_{s c}$ of $23.27 \mathrm{~mA} \mathrm{~cm}^{-2}$, and fill factor (FF) of 0.680 . The better photovoltaic performance of the PN-Se based device is benefitted from its more suitable phase separation, faster exciton dissociation and charge transportation and less charge recombination. These results demonstrate the importance of selecting suitable SMA building block unit in the design of high-performance PSMAs, and provide an effective way to adjust the photoactive layer morphology in the all-PSCs.

\section{Results}

The synthesis of PSMAs PS-Se and PN-Se. Figure 2 shows the chemical structures and the synthetic routes of PS-Se and PN-Se. Both PSMAs were synthesized by Stille cross-coupling polymerization of the brominated Y6-like TPBS-Br unit or brominated Y18-like TPBN-Br unit and the 2,5-bis (trimethylstannyl) selenophene linking bridge-unit (see synthetic details in the 
(a)
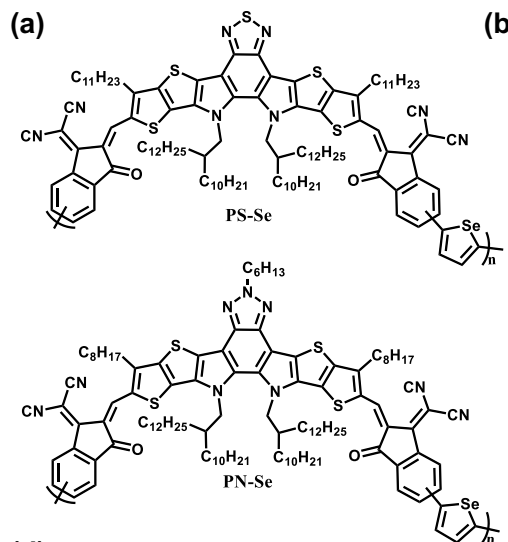

(d)

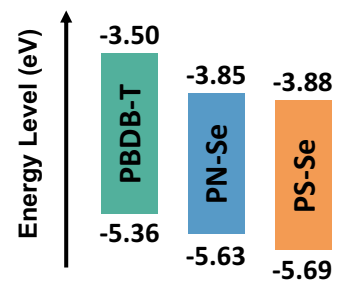

(b)
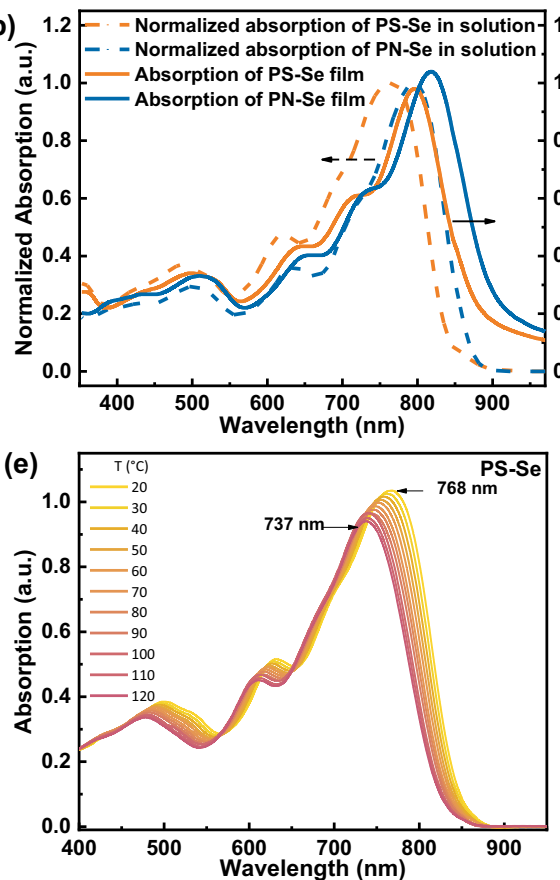

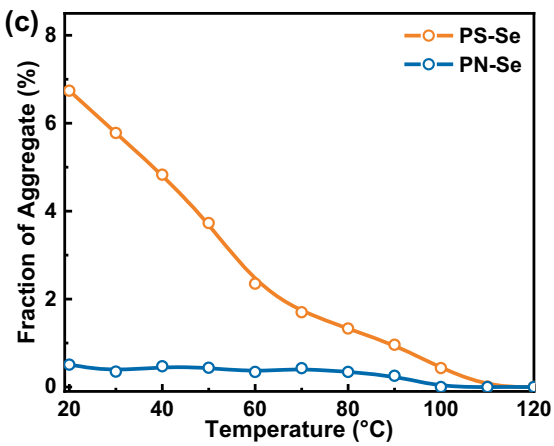

(f)

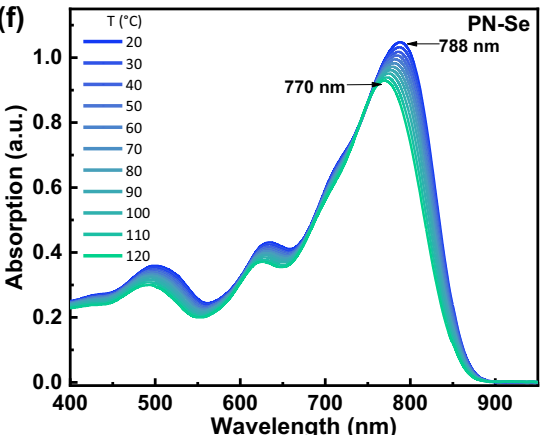

Fig. 1 Molecular structures and physicochemical properties of PS-Se and PN-Se. a Chemical structures of PS-Se and PN-Se acceptors. b UV-vis absorption spectra of PS-Se and PN-Se in chloroform solutions and the corresponding neat films. c Plots of calculated aggregate content (molar \%) of the two polymer acceptors in chlorobenzene at different temperatures. d Energy level diagram of the related materials in the all-PSCs. Variable-temperature UV-vis absorption spectra of (e) PS-Se and (f) PN-Se in chlorobenzene.

"Methods" and "Supplementary methods" sections). To ensure the solubility of the PSMAs PS-Se and PN-Se, longer alkyl side chains were employed in the SMA units. Thus, the resulting polymers can be readily dissolved in common organic solvents at room temperature. In order to reduce the influence of the molecular weights of the polymer acceptors on their physicochemical and photovoltaic properties, two batches of PS-Se and PN-Se were selected with comparable number-average molecular weights $\left(M_{n}\right)$ measured by gel permeation chromatography (GPC), as shown in Supplementary Fig 19. The number-average molecular weight and polydispersity index were $11.5 \mathrm{kDa} / 2.7$ and $11.7 \mathrm{kDa} / 2.8$ for PS-Se and PN-Se, respectively. Thermal stabilities of PS-Se and PN-Se were characterized by thermogravimetric analysis (TGA), and both PSMAs showed good thermal stability with decomposition temperature $\left(T_{\mathrm{d}}, 5 \%\right.$ weight loss $)$ at $343^{\circ} \mathrm{C}$ under nitrogen atmosphere (as shown in Supplementary Fig. 1).

\footnotetext{
Absorption spectra and electronic energy levels of the PSMAs. $\mathrm{UV}$-vis absorption spectra of PS-Se and PN-Se in chloroform solution and neat films were measured to investigate the effect of the $\mathrm{BS}$ and $\mathrm{BN}$ core on the optical and packing properties of the PSMAs. As shown in Fig. 1b, either in chloroform solution or film state, PN-Se shows more red-shifted absorption than PS-Se. In solution, PS-Se and PN-Se both exhibit broadened absorption range from 600 to $900 \mathrm{~nm}$ with maximum peaks at 766 and $796 \mathrm{~nm}$, respectively. In neat films, PS-Se displays an absorption peak at $798 \mathrm{~nm}$ with absorption coefficient of $0.98 \times 10^{5} \mathrm{~cm}^{-1}$, and PN-Se shows a featured peak at $820 \mathrm{~nm}$ with absorption coefficient of $1.04 \times 10^{5} \mathrm{~cm}^{-1}$. It is worth noting that the degree of red-shift for PS-Se and PN-Se films were observed as 32 and $24 \mathrm{~nm}$, respectively, in comparison with its solution. The larger degree of red-shift is associated with more intimate $\pi-\pi$ stacking interaction and aggregation ${ }^{37}$. Since the changes in absorption
}

range and intensity are the signature of aggregation of the polymers, variable-temperature UV-vis absorption spectra were further measured in the temperature range of $120-20^{\circ} \mathrm{C}$ (as shown in Fig. 1e, f). At the high temperature of $120^{\circ} \mathrm{C}$, the polymer chains will be in disordered phase. During the cooling process from the high temperature of $120^{\circ} \mathrm{C}$, a fraction of the polymer chains will be aligned in a well-ordered aggregation while the rest of polymer chains remain disordered ${ }^{38}$, which could affect the overall absorption spectra. This fraction of aggregation $\left(f_{\text {aggr }}\right)$ could be calculated according to the change between the absorption of the pure disordered phase at high temperature $\left(120^{\circ} \mathrm{C}\right)$ and the superposition absorption of disordered and aggregated chains at the lower temperature ${ }^{39}$ (Fig. 1c). From 120 to $20^{\circ} \mathrm{C}$, the PS-Se film exhibits more red-shifted vibronic absorption peak (by $31 \mathrm{~nm}$ from 737 to $768 \mathrm{~nm}$ ) than that of the PN-Se film (by $18 \mathrm{~nm}$ from 770 to $788 \mathrm{~nm}$ ), which should be associated with the stronger aggregation in PS-Se. While the smaller red-shift in vibronic feature of the PN-Se film might also be caused by planarization of the disordered chains rather than aggregation $^{38}$.

Frontier orbital energy levels (HOMO/LUMO) of the PS-Se and PN-Se were estimated from electrochemical cyclic voltammetry (CV) measurements (Fig. 1d). From the onset oxidation potential $\left(E_{\mathrm{Ox}}\right)$ and onset reduction potential $\left(E_{\mathrm{red}}\right)$, the HOMO energy level $\left(E_{\mathrm{HOMO}}\right)$ and the LUMO energy level $\left(E_{\mathrm{LUMO}}\right)$ of the polymers were calculated according to the equation $E_{\mathrm{LUMO} / \mathrm{HOMO}}=-\mathrm{e}\left(E_{\mathrm{red} / \mathrm{ox}}+4.36\right)(\mathrm{eV})$ where the unit of $E_{\text {red/ox }}$ is $\mathrm{V}$ vs. $\mathrm{Ag} / \mathrm{AgCl}$. Redox potential of $\mathrm{Fc} / \mathrm{Fc}^{+}$is $0.44 \mathrm{~V}$ vs. $\mathrm{Ag} / \mathrm{AgCl}$ in our measurement system (see Supplementary Fig. 2), and we took the energy level of $\mathrm{Fc} / \mathrm{Fc}^{+}$as $4.8 \mathrm{eV}$ below vacuum. The $E_{\mathrm{LUMO}} / E_{\mathrm{HOMO}}$ values were estimated to be $-3.88 /-5.69 \mathrm{eV}$ for $\mathrm{PS}-\mathrm{Se}$, and $-3.85 /-5.63 \mathrm{eV}$ for PN-Se, respectively. The electronic energy levels and other physicochemical properties of the polymer acceptors are listed in Supplementary Table 1 for a clear comparison. 

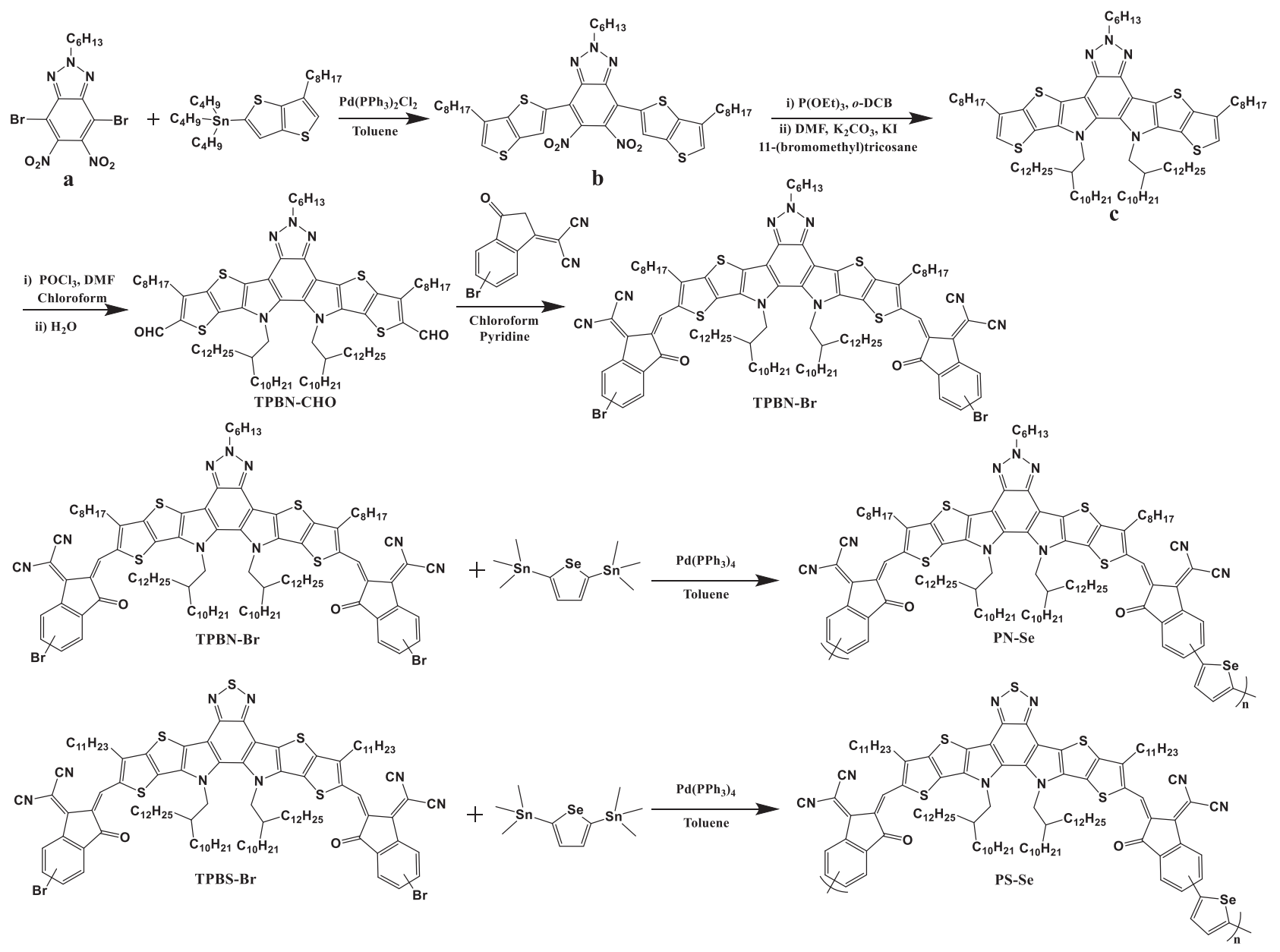

Fig. 2 Synthetic routes of the PSMAs. The synthetic routes of PS-Se and PN-Se.

Visualization of aggregation behavior of the polymer blends in solution by Cryo-TEM. Cryo-TEM is a unique technique to characterize the aggregation and dispersion structures dispersed in liquid suspensions that are close to their native state ${ }^{33,40,41}$. After the rapid freezing of the polymer donor/polymer acceptor blend precursor solutions, the preaggregation state dispersed in the solution could be visualized and characterized by highresolution TEM. As mentioned above, the morphology of the polymer donor/polymer acceptor blend films is mainly controlled by the preaggregation state in their solutions. Hence, we performed the Cryo-TEM measurements to visualize the preaggregation state of the polymer blends in their chloroform solutions. In this work, PBDB-T was used as the polymer donor, and the concentration of $14 \mathrm{mg} \mathrm{mL}^{-1}$ for the total polymer donor and acceptor was adopted in the all-polymer blend solutions (see sample preparation details in "Method" section). Figure 3 shows Cryo-TEM images of the frozen polymer blend chloroform solutions. In the frozen PBDB-T:PN-Se blend solution, the polymers show dark gray thin lines with a uniform aggregation size of ca. $8-16 \mathrm{~nm}$ (see Fig. 3a), which could form the ideal interpenetrating networks morphology in the $\mathrm{BHJ}$ blend films prepared from the solution. While for the PBDB-T:PS-Se blend solution (see Fig. 3b), larger dense blocks of dispersed aggregates were observed, and the reticular aggregates with slightly larger size of ca. $14-23 \mathrm{~nm}$ were more coarsely distributed. The results of the Cryo-TEM indicate that the chemical structures of PSMA plays an important role on the aggregation morphology in the blend solutions. Obviously, the different sizes and shapes of the aggregates of the polymer blends in their solutions would affect the final film morphology prepared from the solution when fabricating the all-PSCs.

Morphological characterizations of polymer films. First, the Flory-Huggins interaction parameters $\left(\chi_{\mathrm{da}}\right)$ were calculated from contact-angle measurements of the neat films. The corresponding surface energy of the polymer donor $\left(\gamma_{\mathrm{d}}\right)$ and acceptors $\left(\gamma_{\mathrm{a}}\right)$ (Supplementary Table 2) could assess the miscibility between the donor and acceptor, according to the equation $\chi_{\mathrm{da}}=K\left(\sqrt{\gamma_{d}}-\sqrt{\gamma_{\mathrm{a}}}\right)^{2}$, where $K$ is a constant ${ }^{42}$. The $\chi_{\mathrm{da}}$ values were $0.30 \mathrm{~K}$ and $0.19 \mathrm{~K}$ for PBDB-T:PS-Se and PBDB-T:PN-Se blend films, respectively. The smaller $\chi_{\mathrm{da}}$ of PBDB-T:PN-Se suggests greater miscibility and is correlated with the increasing density of D/A interfaces in the blend films ${ }^{43}$.

To further investigate the effect of BS core in PS-Se and BN core in $\mathrm{PN}-\mathrm{Se}$ on the morphological characteristics, the morphologies of the PS-Se- and PN-Se-based blend films were measured by atomic force microscopy (AFM) and photo-induced force microscopy (PiFM). Combining the response of photoinduced polarizability of polymer donor or polymer acceptor in the near field and AFM technology, PiFM is capable of distinguishing the donor and acceptor at nanometer scale and providing a thorough and direct compositional analysis of the allpolymer $\mathrm{BHJ}$ films ${ }^{35,44}$. First, we measured infrared (IR) absorption spectra of neat PS-Se and PN-Se films (see the Supplementary Fig. 3), and both films presented several IR 

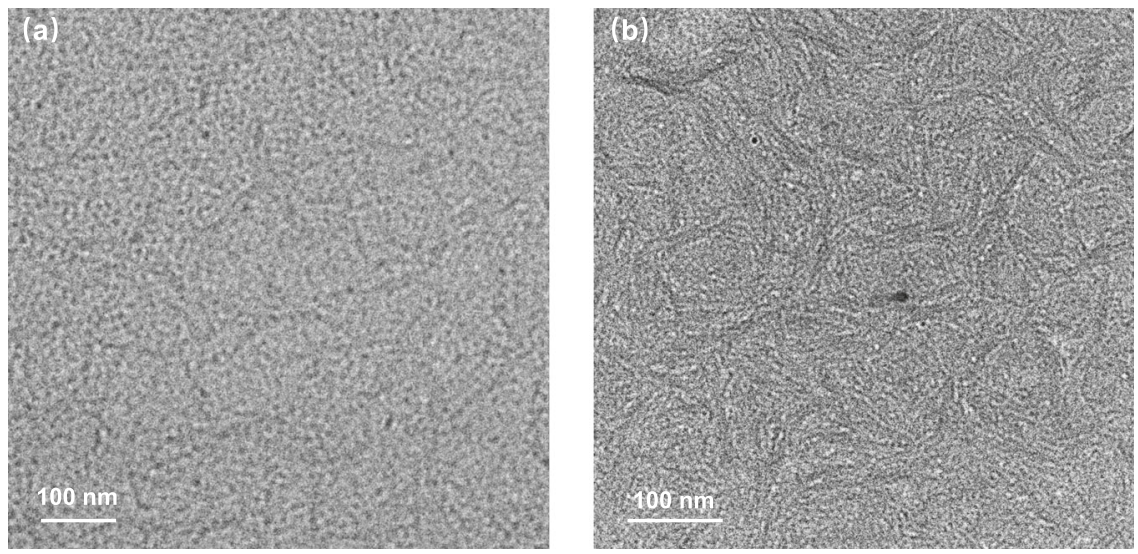

Fig. 3 Cryo-TEM images. a Cryo-TEM images of the frozen PBDB-T:PN-Se blend precursor solution in chloroform. $\mathbf{b}$ Cryo-TEM images of the frozen PBDB$\mathrm{T}: \mathrm{PS}-\mathrm{Se}$ blend precursor solution in chloroform.

(a) (1)

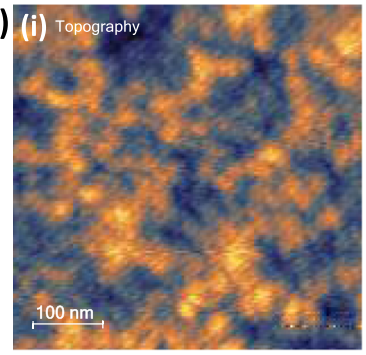

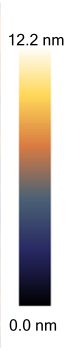
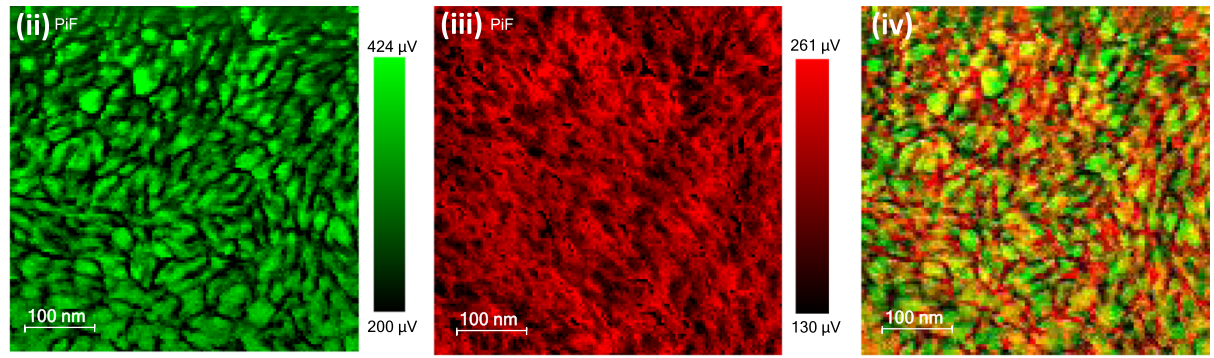

(b) (i)
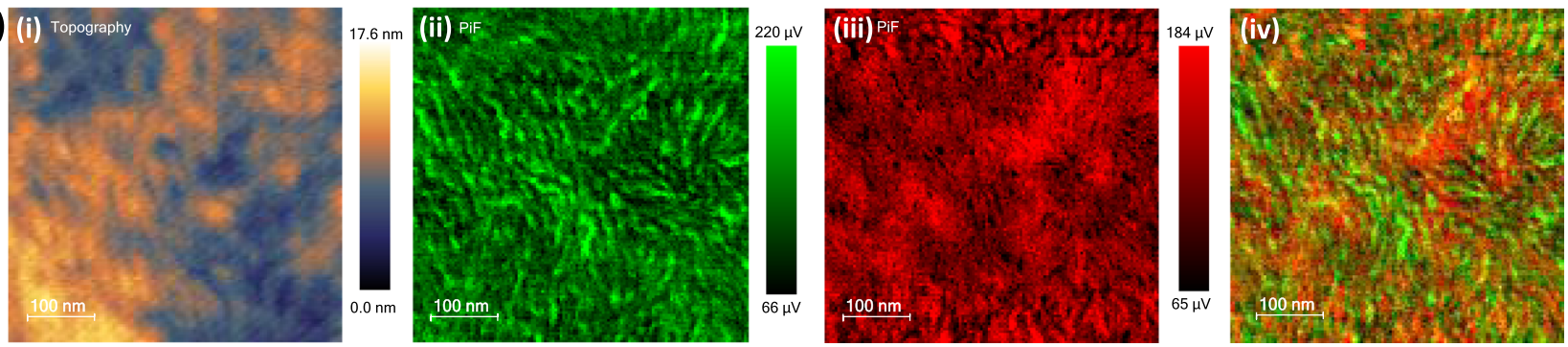

Fig. 4 AFM and PiFM images of the blend films. a Patterns of PBDB-T:PN-Se blend. $\mathbf{b}$ Patterns of PBDB-T:PS-Se blend. (i) AFM topography images of

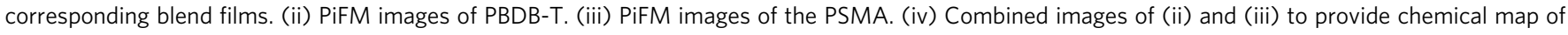
PBDB-T and PSMA.

characteristic peaks. Here, the IR absorption wavenumber of $1653 \mathrm{~cm}^{-1}$ was chosen as the characteristic peak of donor PBDB$\mathrm{T}$ component and $1538 \mathrm{~cm}^{-1}$ as the characteristic peak of acceptor PS-Se or PN-Se component. Figure 4 shows the AFM and PiFM images of the PBDB-T:PS-Se and PBDB-T:PN-Se blend films which are fabricated by the same optimized conditions in their corresponding all-PSCs. As shown in Fig. 4a(i), the AFM topography pattern of PBDB-T:PN-Se blend film displays distinct and uniform phase separation with small fibrous aggregates. In particular, the PiFM images of PBDB-T (green color, as shown in Fig. $4 a(i i))$ and PN-Se (red color, as shown in Fig. 4a(iii)) all show more distinct fibrous network. After combining the PiFM images of PBDB-T and PN-Se in Fig. 4a(iv), the blend film exhibits an unique $\mathrm{BHJ}$ bicontinuous-interpenetrating network of polymer donor/polymer acceptor with PN-Se polymer acceptor surrounding the PBDB-T polymer donor region. The domain size of the distinguishable phase separation was estimated to be $10-20 \mathrm{~nm}$, which is beneficial for exciton dissociation and charge transportation ${ }^{45}$ and could lead to higher photovoltaic performance of the all-PSCs. In contrast, PBDB-T:PS-Se blend film (Fig. 4b(iv)) shows bigger donor domain (green color) and acceptor domain (red color) with the oversized phase separation over $30 \mathrm{~nm}$ of PBDB-T and PS-Se, which is consistent with the stronger aggregation of PS-Se confirmed from the variabletemperature UV-vis absorption and the Cryo-TEM analysis mentioned above. In general, the ideal phase separation scale should be around $20 \mathrm{~nm}^{46}$. The oversized phase separation decreases the interface areas in the $\mathrm{BHJ}$ films, which has negative effect on exciton dissociation and charge separation, and would influence the photovoltaic performance ${ }^{11}$.

Similarly, the AFM topography image (Fig. 4a(i)) of PBDBT:PN-Se shows smoother surface with smaller values of rootmean-square (RMS) roughness of $1.67 \mathrm{~nm}$, compared with the PBDB-T:PS-Se blend film (Fig. 4b(i)) with RMS of $2.21 \mathrm{~nm}$. The smoother surface could favorably improve the contact between the interfacial layer and the active layer ${ }^{47}$. Besides, the TEM images, as shown in Supplementary Fig. 4, exhibit similar phase separation tendency to the PiFM images.

In addition to phase separation size, the crystallinity and polymer chain arrangement also influence the exciton diffusion, exciton dissociation and free charge carrier transport. Thus, we further conducted grazing-incidence wide-angle X-ray scattering 

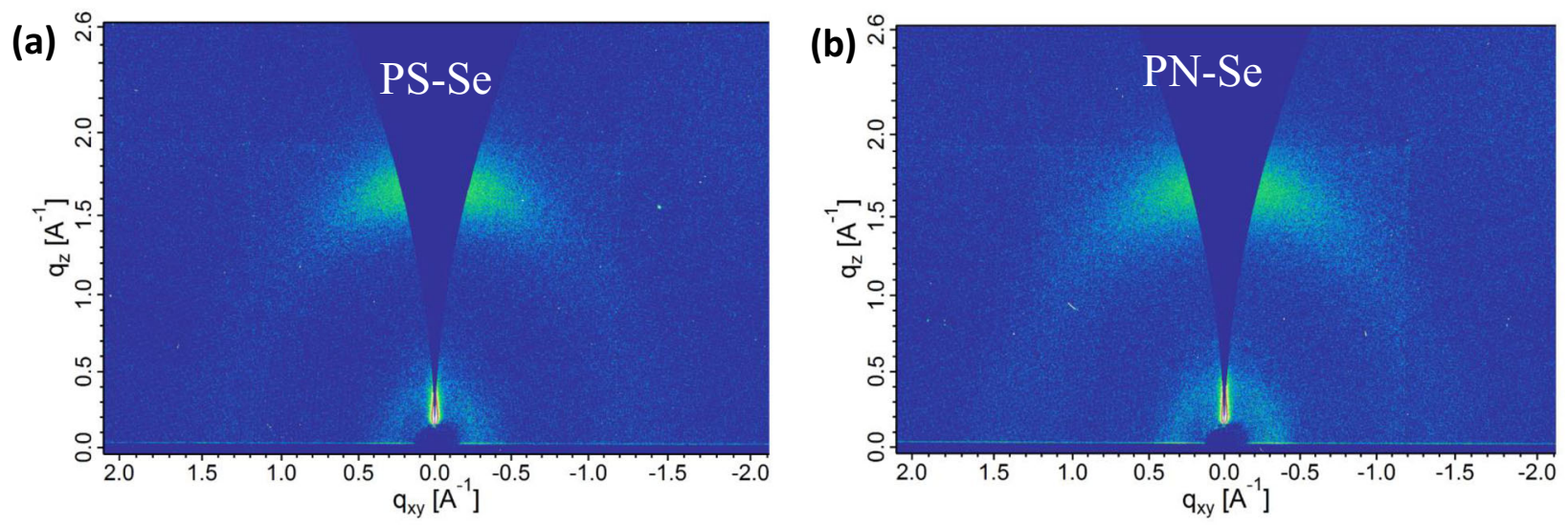

(c)

(d)
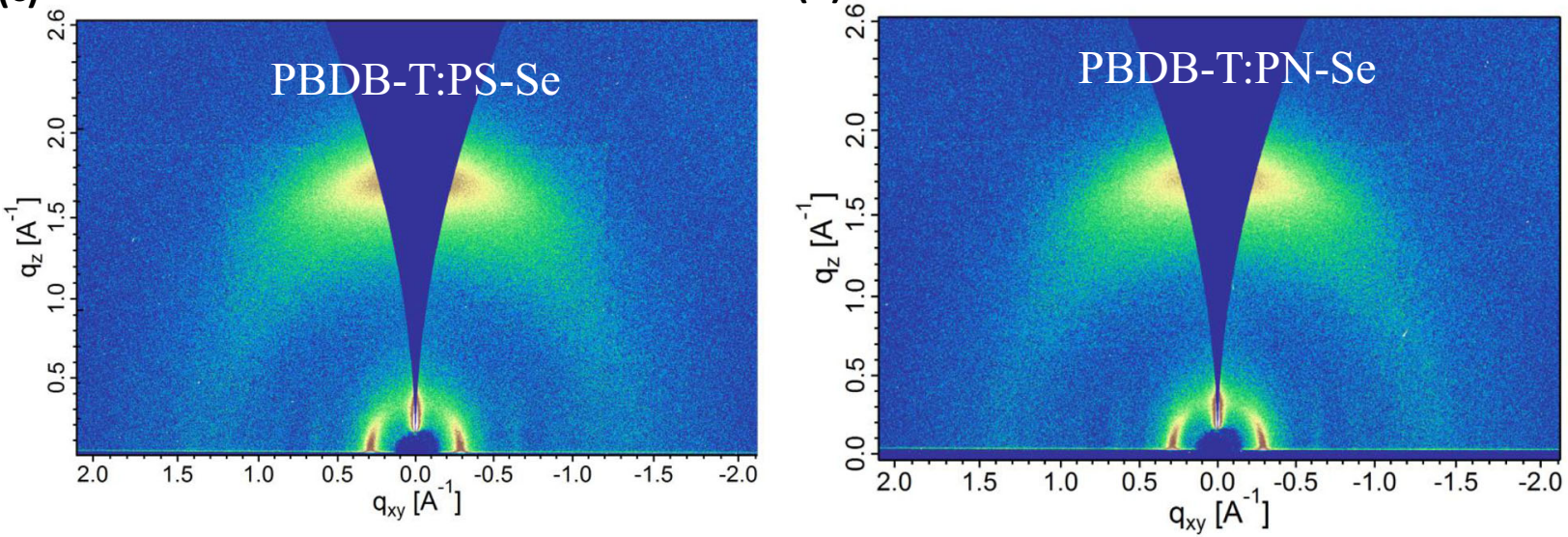

Fig. 5 2D-GIWAXS scattering patterns. 2D-GIWAXS scattering patterns of PS-Se and PN-Se neat films (a, b), and their blend films (c, d) with polymer donor PBDB-T

(GIWAXS) measurements ${ }^{48}$. Figure $5 \mathrm{a}, \mathrm{b}$ shows the twodimensional (2D) patterns and Supplementary Fig. 5 a, b shows the one-dimensional (1D) extracted profiles of the neat films. PSSe and PN-Se neat films display clear (010) peaks in out-of-plane (OOP) direction and (100) peak in in-plane (IP) direction, suggesting that both acceptors contain preferred face-on orientations. Comparatively, PS-Se shows (010) peak at $q=1.65 \AA^{-1}$ $\left(d_{(010)}=3.81 \AA\right)$ while PN-Se at $q=1.66 \AA^{-1}\left(d_{(010)}=3.78 \AA\right)$, and the corresponding crystal coherence lengths (CCLs) of (010) orientation are 19.1 and $16.5 \AA$ calculated by Scherrer equation for PS-Se and PN-Se, respectively. Besides, for the blend films, the 2D-GIWAXS scattering patterns and the corresponding 1D inand out-of-plane sector cuts are shown in Fig. 5c, d and Supplementary Fig. 5 c, d, respectively, and the relevant molecular packing parameters are summarized in Supplementary Table 3 and 4. Overall, all the blend films maintain the clear face-on orientation. To better understand the differences between PBDBT:PS-Se and PBDB-T:PN-Se, the molecular packing and CCLs of the individual donor and acceptor in the blends were investigated by deconvoluting the (010) and (100) peaks to its individual donor and acceptor components. For the donor PBDB-T, the CCL value of the (010) peak in the PBDB-T:PS-Se blend film is 28.6 $\AA$, which is slightly larger than $27.3 \AA$ in the PBDB-T:PN-Se blend film. In general, longer (010) CCL implies better molecular ordering along the $\pi-\pi$ packing orientation of the PBDB-T:PS-Se film. On the other hand, for the polymer acceptor, both PBDBT:PS-Se and PBDB-T:PN-Se blend films show similar (100) peak at $q=0.28 \AA^{-1}\left(d_{(100)}=22.4 \AA \mathrm{CCL}=31.4 \AA\right)$ and $(010)$ peak at $q=1.65 \AA^{-1}\left(d_{(010)}=3.81 \AA \mathrm{CCL}=11.6 \AA\right)$, indicating that there is no much difference of the $\pi-\pi$ stacking distances and the coherences across the films between the two polymer blend active layers. However, the integrated intensity (normalized to the thickness (Volume) and the background) of the $\pi-\pi$ stacking (010) peak and lamellar stacking (100) peak are marginally higher for both the acceptor and the donor components of the PBDB$\mathrm{T}: \mathrm{PN}-\mathrm{Se}$ blend, suggesting a favorably oriented and more ordered arrangement of the PN-Se and PBDB-T grains for its better charge transport properties. The relatively higher diffracted intensity can be interpreted as a smaller volume fraction of disordered material ${ }^{49}$. Additionally, the corresponding $g$ parameters $^{50}$ were calculated for PBDB-T:PS-Se $(g=14.3$ for donor and $g=22.8$ for acceptor) and PBDB-T:PN-Se $(g=14.6$ for donor and 22.8 for acceptor), indicating the material is amorphous in the $\pi-\pi$ stacking direction of the polymers ${ }^{51}$. In this case, the ordered arrangement of the aggregates and presence of interconnected morphology such as better bicontinuousinterpenetrating polymer donor/polymer acceptor networks are more important for charge separation and transportation.

Photovoltaic performance of the all-PSCs. The photovoltaic performance of the PSMA polymer acceptors were studied by fabricating the all-PSCs with the conventional device structure of ITO/PEDOT:PSS/PBDB-T:PSMA/PDINN $52 / A g$. The donor to acceptor weight ratios were fixed at the optimized weight ratio of $1.5: 1$, and the current density-voltage $(J-V)$ curves of the all-PSCs were measured under the illumination of AM1.5 G, $100 \mathrm{~mW} \mathrm{~cm}^{-2}$. Detailed processes of fabricating the all-PSCs are described in "Method" section. Figure 6a shows the optimal $J-V$ curves and Table 1 lists the photovoltaic parameters of the devices. The 

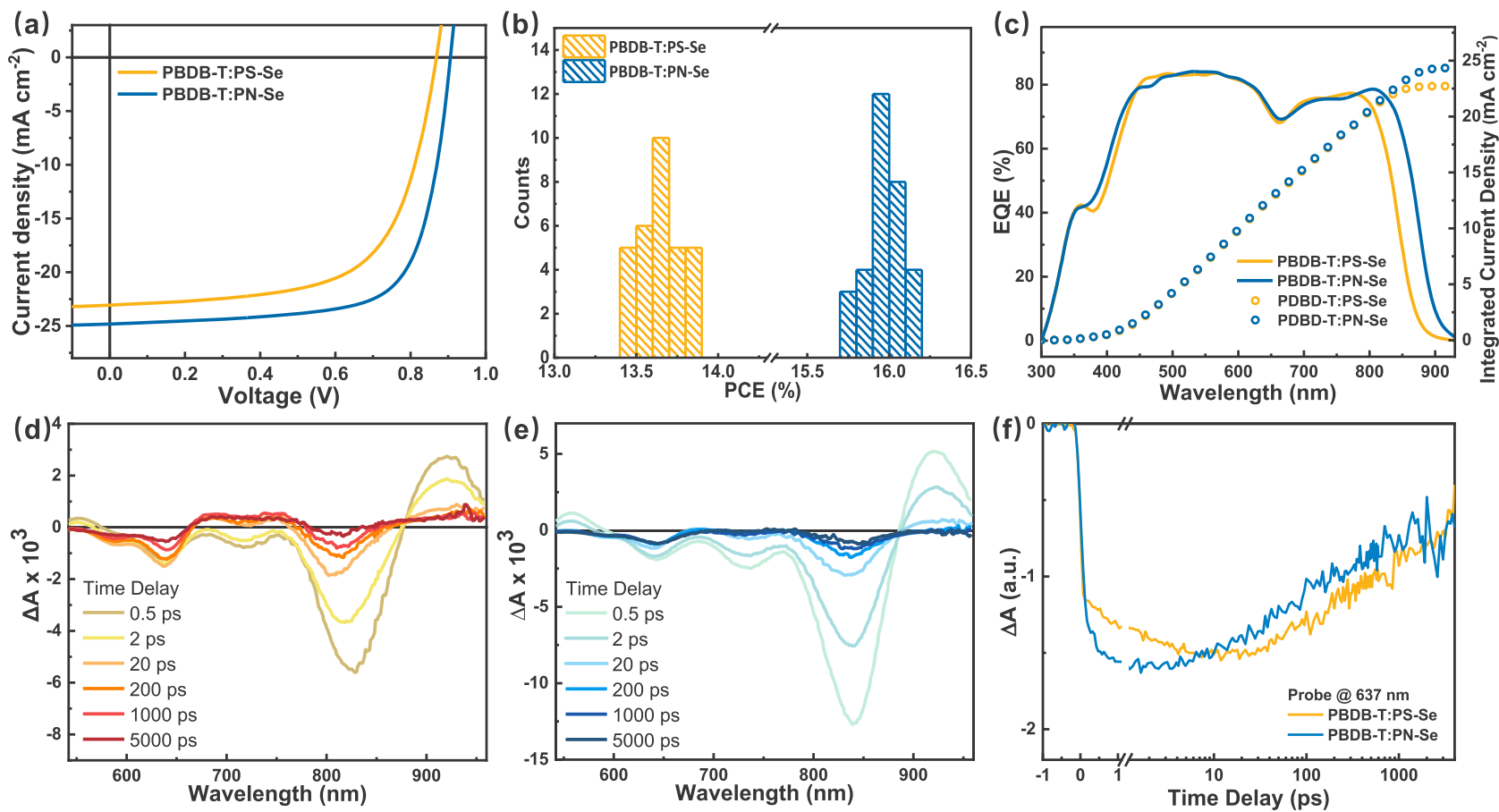

Fig. 6 Photovoltaic performance and transient absorption kinetics. a The J-V curves of the optimal all-PSCs under the illumination of AM1.5 G, $100 \mathrm{~mW} \mathrm{~cm}^{-2}$. b Histogram of PCE measurement for 30 devices based on PBDB-T:PS-Se (1.5:1, w/w) and 30 devices based on PBDB-T:PN-Se (1.5:1, w/W), measured under the illumination of $A M 1.5 \mathrm{G}, 100 \mathrm{~mW} \mathrm{~cm}^{-2}$. c External quantum efficiency (EQE) spectra of the corresponding all-PSCs. Femtosecond transient absorption spectra of (d) PBDB-T:PS-Se and (e) PBDB-T:PN-Se with excitation at $850 \mathrm{~nm}$. $\mathbf{f}$ Transient kinetic traces of PBDB-T GSB probing at $637 \mathrm{~nm}$ for the blend films.

\section{Table 1 Photovoltaic parameters of the optimal all-PSCs based on PBDB-T:PSMAs (1.5:1, w/w), measured under the illumination of AM1.5 G, $100 \mathrm{~mW} \mathrm{~cm}^{-2}$.}

\begin{tabular}{|c|c|c|c|c|}
\hline Active layer & $V_{o c}(V)$ & $J_{\mathrm{sc}}\left(\mathrm{mA} \mathrm{cm} \mathrm{cm}^{-2}\right)$ & $\mathbf{F F}$ & PCE (\%) \\
\hline PBDB-T:PS-Se & $\begin{array}{l}0.874 \\
(0.874 \pm 0.003)\end{array}$ & $\begin{array}{l}23.27 \\
(22.84 \pm 0.37)\end{array}$ & $\begin{array}{l}0.680 \\
(0.693 \pm 0.009)\end{array}$ & $\begin{array}{l}13.83 \\
(13.64 \pm 0.12)^{a}\end{array}$ \\
\hline PBDB-T:PN-Se & $\begin{array}{l}0.907 \\
(0.907 \pm 0.003)\end{array}$ & $\begin{array}{l}24.82 \\
(24.73 \pm 0.26)\end{array}$ & $\begin{array}{l}0.718 \\
(0.713 \pm 0.005)\end{array}$ & $\begin{array}{l}16.16 \\
(15.97 \pm 0.13)^{a}\end{array}$ \\
\hline
\end{tabular}

all-PSC based on PBDB-T:PS-Se showed a PCE of $13.83 \%$ with a $V_{o c}$ of $0.874 \mathrm{~V}, J_{s c}$ of $23.27 \mathrm{~mA} \mathrm{~cm}^{-2}$, and FF of 0.680 . As expected, the PBDB-T:PN-Se based all-PSC achieved an even better PCE of $16.16 \%$ with a $V_{o c}$ of $0.907 \mathrm{~V}, J_{s c}$ of $24.82 \mathrm{~mA} \mathrm{~cm}^{-2}$, and FF of 0.718 . In comparison with the PS-Sebased device, the accountable reasons for the higher PCE of the $\mathrm{PN}$-Se-based all-PSC are the further increased $V_{o c}$ benefited from the higher lying LUMO energy level of the PN-Se polymer acceptor, the enhanced $J_{s c}$ resulted from the red-shifted absorption and higher absorption coefficient of PN-Se, and the better FF benefitted from the well-formed blend morphology of the PBDB-T:PN-Se blend active layer. Figure $6 \mathrm{~b}$ further shows the PCE histogram obtained from the PBDB-T:PS-Se- and PBDB$\mathrm{T}$ :PN-Se-based devices, indicating the better reproducibility of the high photovoltaic performance of the PBDB-T:PN-Se-based all-PSCs. In addition, Supplementary Table 7 lists the molecular weights of PS-Se and PN-Se and the PCEs of the all-PSCs with PBDB-T polymer donor and the PSMA polymer acceptors with different molecular weights. It can be seen that for the different batches of PSMA acceptors in the molecular weight range of 8-12 kDa, all the PN-Se-based all-PSCs show higher PCE than that of the PS-Se-based devices.
The external quantum efficiency (EQE) of the PBDB-T:PS-Seand PBDB-T:PN-Se-based devices were measured and shown in Fig. 6c. Both all-PSC devices show broad and stronger photoresponse from 300 to $900 \mathrm{~nm}$, while the PN-Se-based allPSC displayed a broader photoresponse extending to over $900 \mathrm{~nm}$, benefitted from the red-shifted absorption of PN-Se. The $J_{\mathrm{sc}}$ values integrated from the EQE values are 22.72 and $24.36 \mathrm{~mA} \mathrm{~cm}^{-2}$ for the PS-Se- and PN-Se-based devices, respectively. Both the integrated $J_{\mathrm{sc}}$ values are in good agreement with those values obtained from the $J-V$ characteristics of the all- PSCs.

Thermal stability is one of the major advantages of all-PSCs. We examined the thermal stability of the active layer films based on PS-Se and PN-Se. The active layers were annealed at $150^{\circ} \mathrm{C}$ for different times before fabricating into all-PSC devices. After $600 \mathrm{~min}$ of thermal annealing stress, photovoltaic performance of the PS-Se and PN-Se based all-PSCs did not show significant degradation (as shown in Supplementary Fig. 6). The results prove that the blend films based on PS-Se and PN-Se have good thermal stability under the harsh test conditions.

We further carried out the studies on the photo-induced charge transfer (CT) process of the active layer by measuring the femtosecond transient absorption spectra (fs-TA) of the blend 
films of PBDB-T:PS-Se and PBDB-T:PN-Se ${ }^{53}$. Pump wavelength was set to $850 \mathrm{~nm}$ to selectively excite the polymer acceptors in the blend films. Figure $6 \mathrm{~d}$ shows the fs-TA spectra of the PBDBT:PS-Se blend at the selected time delays. Photoexcitation of the acceptor leads to the formation of PS-Se excitons in the film, a broad ground state bleach (GSB) peak at $825 \mathrm{~nm}$ was observed that matches the absorption spectrum of PS-Se. Additional GSB was also apparent at $637 \mathrm{~nm}$, which is attributed to the donor PBDB-T, resulted from the ultrafast hole transfer from PS-Se to PBDB-T directly at the D/A interface. With the decay of PS-Se excited state, the intensity of the GSB of PBDB-T gradually grows over the subsequent $20 \mathrm{ps}$, indicating a continuous hole transfer process. The GSB of PS-Se and PBDB-T then decays concomitantly, since the CT state starts to recombine to ground state. Similar transient absorption spectra were also observed for the PBDB-T:PN-Se blend except for the red-shifted GSB of PN-Se (Fig. 6e). The results agree well with the high EQE values of both all-PSCs based on PBDB-T:PS-Se and PBDB-T:PN-Se.

Then we monitored the kinetic trace of the GSB of PBDB-T for both blends, the hole transfer dynamics at $637 \mathrm{~nm}$ were extracted in Fig. 6f. The PN-Se based blend exhibited a faster hole transfer rate than that of PS-Se. We further investigated the carrier transport properties by measuring the charge carriers mobility with the space-charge-limited-current (SCLC) method. As shown in Supplementary Fig. 7 and Supplementary Table 8, the average electron mobilities of ten devices based on PBDB-T:PS-Se and PBDB-T:PN-Se were estimated to be $(4.84 \pm 0.48) \times 10^{-4}$ and $(6.87 \pm 0.50) \times 10^{-4} \mathrm{~cm}^{2} \mathrm{~V}^{-1} \mathrm{~s}^{-1}$, respectively. And the average hole mobilities of ten devices based on PBDB-T:PS-Se and PBDB$\mathrm{T}: \mathrm{PN}-\mathrm{Se}$ were estimated to be $(6.89 \pm 0.52) \times 10^{-4}$ and $(7.92 \pm 0.49) \times 10^{-4} \mathrm{~cm}^{2} \mathrm{~V}^{-1} \mathrm{~s}^{-1}$, respectively. The electron and hole mobilities of the PN-Se-based blend film are higher than those of the PS-Se-based blend film. For the PN-Se-based blend, its higher hole mobility agrees with the hole transfer dynamics results shown in Fig. $6 \mathrm{f}$ and could be benefitted from the ideal phase separation and better D/A interpenetrating structure in its blend films as mentioned above. Moreover, the smaller $\mu_{\mathrm{h}} / \mu_{\mathrm{e}}$ ratio (1.15) in the PBDB-T:PN-Se blend film indicates more balanced electron/hole transport capability of the PBDB-T:PN-Se blend film. The higher and more balanced electron/hole mobilities could be responsible to the higher FF of the PN-Se based all-PSCs ${ }^{54}$.

To further evaluate the carrier trapping and detrapping in the all-polymer blend, we measured the transient photocurrent (TPC). The photocurrent curves of the PS-Se- and PN-Se-based devices all displayed little change with light intensity up to 1 sun equivalent (as shown in Supplementary Fig. 8a, b). In details, as the light intensity increasing from 0.1 to 1 sun, the decay times shortened from 0.40 to $0.34 \mu$ s for the PS-Se based devices and from 0.34 to $0.25 \mu$ s for the PN-Se-based devices (see Supplementary Fig. 8c). In general, the faster rise/fall behavior of photocurrent and shorter transport time $\left(\tau_{\mathrm{t}}\right)$ indicate favorable charge carrier transport, less electron trap states and faster transport-limiting recombination ${ }^{55}$. Furthermore, transient photovoltage (TPV) decays were measured to estimate the charge carrier lifetimes $(\tau)$. The carrier lifetimes of the PN-Se based device are consistently longer than the PS-Se-based device across the entire light intensity range from 0.1 to 1 sun (as shown in Supplementary Fig. 8d), which indicates less carrier recombination ${ }^{46}$. All the results mentioned above are matched well with the morphology analysis and the excellent photovoltaic performance of the PN-Se-based all-PSCs.

\section{Discussion}

In this work, we designed and synthesized two new PSMAs PS-Se and PN-Se, based on A-DA'D-A structured Y6-like SMA with benzo[c] $[1,2,5]$ thiadiazole (BS) A'-core or Y18-like SMA with benzotriazole $(\mathrm{BN}) \mathrm{A}^{\prime}$-core as building blocks, polymerized with selenophene as $\pi$-bridge linking unit. The analysis from Variabletemperature UV-vis absorption and Flory-Huggins interaction parameters $\left(\chi_{\mathrm{da}}\right)$ indicate that the $\mathrm{PN}-\mathrm{Se}$ shows suitable aggregation and better miscibility with PBDB-T polymer donor, compared to PS-Se, leading to the desirable phase separation in the polymer blend film. More importantly, the Cryo-TEM images of the frozen polymer blend solutions confirm the ideal polymer donor/polymer acceptor interpenetrating networks with phase separation size of ca. $8-16 \mathrm{~nm}$ in the preaggregation state of the PBDB-T:PN-Se blend solution. The results of PiFM analysis further clearly reveal that the PBDB-T:PN-Se blend exhibits distinct bicontinuous-interpenetrating polymer donor/polymer acceptor networks, which improves the exciton dissociation and charge transportation in the all-PSC. In addition, GIWAXS results indicate a more ordered and popularly oriented arrangement of the PN-Se-based blend film than that of PS-Se. Eventually, the PBDB-T:PN-Se-based all-PSC demonstrates the higher PCE of $16.16 \%$, with a preferable $V_{o c}$ of $0.907 \mathrm{~V}, J_{s c}$ of $24.82 \mathrm{~mA} \mathrm{~cm}^{-2}$, and FF of 0.718 . This work not only realizes a highly efficient all-PSC based on a high-performance polymer acceptor PN-Se, but also provides a clear understanding of the relationship among the molecular structure, nanoscale morphology, photophysical properties, and photovoltaic performance, which could provide insights for the future molecular design of PSMAs and device optimization of the all-PSCs.

\section{Methods}

Materials and synthesis. All chemicals and solvents were purchased from Innochem, J\&K, Alfa Aesar, and TCI Chemical Co., respectively. Other common materials and chemical reagents were purchased from commercial sources and used as received. Toluene was distilled from sodium and benzophenone under nitrogen before using. Polymer donor PBDB-T was purchased from Solarmer Materials Inc., and TPBS-Br was purchased from Hyper Inc.

The synthetic routes of the conjugated polymer acceptors PS-Se and PN-Se are shown in Fig. 2. The precursor a was synthesized by a simple nitration of 4,7dibromo-2-hexyl-2H-benzo[d][1,2,3]triazole. The detailed synthetic procedures and characterizations of the chemical structures of the other monomers and the polymer acceptors are described in the Supplementary method section. The NMR spectra of the compounds are included in Supplementary Fig. 9 18.

Material characterization. ${ }^{1} \mathrm{H}-\mathrm{NMR}$ and ${ }^{13} \mathrm{C}-\mathrm{NMR}$ spectra were measured on a Bruker DMX-400 spectrometer with $d$-chloroform as the solvent and trimethylsilane as the internal reference. While for the PSMA PS-Se and PN-Se, ${ }^{1} \mathrm{H}$-NMR spectra were measured with 1,1,2,2,-Tetrachlorethane-D2 as solvent at $80^{\circ} \mathrm{C}$. Mass spectra measurement was performed on a Shimadzu spectrometer. TGA was conducted under a nitrogen flow rate of $100 \mathrm{~mL} \mathrm{~min}^{-1}$ on a PerkinElmer TGA-7 thermogravimetric analyzer at a heating rate of $20^{\circ} \mathrm{C} \mathrm{min}-1$. UV-visible absorption spectra and Variable-temperature UV-vis absorption spectra were measured on a Hitachi U-3010 UV-vis spectrophotometer. The electrochemical cyclic voltammetry was carried out on a Zahner IM6e Electrochemical Workstation, in an acetonitrile solution of $0.1 \mathrm{~mol} \mathrm{~L}^{-1} n-\mathrm{Bu}_{4} \mathrm{NPF}_{6}$ at a potential scan rate of $100 \mathrm{mV} \mathrm{s}^{-1}$. The sample film on Pt plate was used as working electrode, a platinum wire was used as counter electrode and $\mathrm{Ag} / \mathrm{AgCl}$ was used as reference electrode. The ferrocene/ferrocenium $\left(\mathrm{Fc} / \mathrm{Fc}^{+}\right)$couple was used as an internal reference. Gel permeation chromatography (GPC) measurement was performed on Agilent PL-GPC 220 instrument with high temperature chromatograph, using $1,2,4$-trichlorobenzene as the eluent at $160^{\circ} \mathrm{C}$.

Cryo-TEM measurements. The samples for the Cryo-TEM observation were prepared with the Vitrobot Mark IV (ThermoFisher Scientific). Before the preparation of samples, we put the grid in the Vitrobot Mark IV at room temperature which is the same temperature as that of the glove box where we fabricate the allPSCs devices. After the Vitrobot Mark IV maintaining at room temperature (rt) for $30 \mathrm{~min}, 3 \mu \mathrm{l}$ chloroform solution dispersion $\left(14 \mathrm{mg} \mathrm{ml}^{-1}, \mathrm{rt}\right)$ was dripped onto a holey carbon film on copper grid (GIG) and blotted with a piece of filter paper to obtain a thin liquid film on the grid. Then the grid was quickly plunged into liquid ethane. (At this time, the morphology of the polymer donor and PSMA had been frozen.) The vitrified samples were then transferred to a 626 cryogenic sample holder (Gatan) and examined with a Cryo-TEM (Themis 300, ThermoFisher Scientific) at $77 \mathrm{~K}$. Micrographs were captured with a Falcon III camera (ThermoFisher Scientific). 
Device fabrication and characterization. The all-polymer solar cells were fabricated with a structure of ITO/PEDOT:PSS $(40 \mathrm{~nm}) /$ active layer/PDINN/Ag. A thin layer of PEDOT:PSS was deposited on precleaned ITO-coated glass through spin coating a PEDOT:PSS aqueous solution (Baytron P VP AI 4083 from H.C. Starck) at $4000 \mathrm{rpm}$ and dried subsequently at $150^{\circ} \mathrm{C}$ for $15 \mathrm{~min}$ in air, then the PEDOT:PSS-coated ITO glass was transferred to a nitrogen glove box. The PBDBT:polymer acceptors $(1.5: 1, \mathrm{w} / \mathrm{w})$ were dissolved in chloroform (the total concentration of blend solutions was $14 \mathrm{mg} \mathrm{mL}^{-1}$ for all blends), with the addition of $1.5 \mathrm{vol} \% 1$-chloronaphthalene $(\mathrm{CN})$ as additive, and stirred overnight in a nitrogenfilled glove box. The blend solutions were spin-cast onto the PEDOT:PSS layer at a spin-coating rate of $3500 \mathrm{rpm}$ for 30 seconds. After the active layers were treated with thermal annealing, the methanol solution of PDINN with a concentration of $1.0 \mathrm{mg} \mathrm{mL}^{-1}$ was spin-coated atop the active layer at $3000 \mathrm{rpm}$ for 30 seconds to form a cathode buffer layer. Finally, top Ag electrode was deposited in vacuum onto the cathode buffer layer under high vacuum. The active area of the PSCs was $5.0 \mathrm{~mm}^{2}$, which was defined by Optical microscope (Olympus BX51). In order to accurately measure the photocurrent, mask with an area of $4.80 \mathrm{~mm}^{2}$ was used to define the effective area of the OSCs. The devices with or without mask showed consistent photovoltaic performance values with relative errors within $0.3 \%$

The current density-voltage $(J-V)$ curves of the all-PSCs were measured on Keithley 2450 Source-Measure Unit in a glove box filled with nitrogen (oxygen and water contents are smaller than $0.1 \mathrm{ppm}$ ). And the measurements were performed by scanning voltage from -1.5 to $2 \mathrm{~V}$ with a voltage step of $10 \mathrm{mV}$ and delay time of $1 \mathrm{~ms}$. Oriel Sol3A Class AAA Solar Simulator (model, Newport 94023 A) with a $450 \mathrm{~W}$ xenon lamp and an air mass (AM) 1.5 filter was used as the light source, and the light intensity was calibrated to $100 \mathrm{~mW} \mathrm{~cm}^{-2}$ by a Newport Oriel $91150 \mathrm{~V}$ reference cell. Solar Cell Spectral Response Measurement System QE-R3-011 (Enli Technology Co., Ltd., Taiwan) was used to measure the EQE. The light intensity at different wavelength was calibrated with a standard single-crystal Si photovoltaic cell.

Photo-induced force microscopy (PiFM) and atom force microscopy. The PiFM microscope including AFM microscope is from a VistaScopefrom Molecular Vista, Inc., operated in dynamic mode using commercial gold-coated silicon cantilevers (NCHAu) from Nanosensors. The excitation laser for the PiFM measurements is a LaserTune IR Source from Block Engineering.

Grazing incident wide-angle X-ray scattering (GIWAXS). Grazing-incidence wide-angle X-ray scattering (GIWAXS) measurements were conducted at Advanced Light Source (ALS), Lawrence Berkeley National Laboratory, Berkeley, $\mathrm{CA}$ at the beamline 7.3.3. Data were acquired at the critical angle $(0.130)$ of the film with a hard X-ray energy of $10 \mathrm{keV}$. X-ray irradiation time was 30-60 s, dependent on the saturation level of the detector. Beam center was calibrated using $\mathrm{AgB}$ powder and the sample-to-detector distance was about $280 \mathrm{~mm}$. The $\pi-\pi$ coherence lengths $(L)$ are estimated based on the Scherrer Equation $(L=2 \pi \mathrm{K} / \mathrm{FWHM})$, where $\mathrm{K}$ is the shape factor (here we use 0.9 ), and FWHM is the full width at half maximum of the (010) diffraction peaks.

Measurement of charge carrier mobilities. The charge carrier mobilities were measured with the device structure of ITO/PEDOT:PSS/active layer/ $/ \mathrm{MoO}_{3} / \mathrm{Ag}$ for hole mobility and ITO/ZnO/active layer/PDINO/Al for electron mobility. The hole and electron mobilities are calculated according to the SCLC method equation: $J=9 \mu \varepsilon_{\mathrm{r}} \varepsilon_{0} V^{2} / 8 d^{3}$, where $J$ is the current density, $\mu$ is the hole or electron mobility, $V$ is the internal voltage in the device, $\varepsilon_{\mathrm{r}}$ is the relative dielectric constant of active layer material, $\varepsilon_{0}$ is the permittivity of empty space, and $d$ is the thickness of the active layer.

\section{Transient absorption spectroscopy. A Yb: KGW amplifier (PHAROS, Light} Conversion,) supplied laser beams centered at $1030 \mathrm{~nm}$ with pulse duration of $\sim 180 \mathrm{fs}$, pulse repetition rate of $33 \mathrm{kHz}$, and a maximum pulse energy of $0.3 \mathrm{~mJ}$. The output of the amplifier was split into two streams of pulses. One was used to drive an optical parametric amplifier (ORPHEUS-HP, Light Conversion) to obtain the pump beam. Residual stream was directed into an ultrafast spectroscopic system (HARPIA-TA, Light Conversion) to generate the white light continuum probe beam. In the spectrometer, the pump chopped at $150 \mathrm{~Hz}$ frequency was spatially and temporally overlapped with the probe beam on the sample. Pump wavelength was set to $850 \mathrm{~nm}$ to selectively excite the polymer acceptors. Excitation energy of the pump pulse was set to $2 \mu \mathrm{J} / \mathrm{cm}^{2}$ to avoid singlet-singlet annihilation. The film samples for the TA measurements were prepared by spin coating the corresponding materials on quartz plates with $1 \mathrm{~mm}$ thick. The TA samples were annealed in nitrogen atmosphere at $120^{\circ} \mathrm{C}$ for 5 min prior to measurement.

Reporting summary. Further information on research design is available in the Nature Research Reporting Summary linked to this article.

\section{Data availability}

The data that support the findings of this study are available from the corresponding author on request.
Received: 8 April 2021; Accepted: 19 August 2021;

Published online: 06 September 2021

\section{References}

1. Gao, L. et al. All-polymer solar cells based on absorption-complementary polymer donor and acceptor with high power conversion efficiency of $8.27 \%$. Adv. Mater. 28, 1884-1890 (2016)

2. Kim, T. et al. Flexible, highly efficient all-polymer solar cells. Nat. Commun. 6, 8547 (2015).

3. Fan, Q. et al. Mechanically robust all-polymer solar cells from narrow band gap acceptors with hetero-bridging atoms. Joule 4, 658-672 (2020).

4. Diao, Y. et al. Flow-enhanced solution printing of all-polymer solar cells. Nat Commun. 6, 7955 (2015).

5. Sun, H., Guo, X. \& Facchetti, A. High-performance n-type polymer semiconductors: applications, recent. Dev. Chall. Chem. 6, 1310-1326 (2020).

6. Zhang, Z.-G. et al. Constructing a strongly absorbing low-bandgap polymer acceptor for high-performance all-polymer solar cells. Angew. Chem. Int. Ed. 56, 13503-13507 (2017)

7. Zhang, Z.-G. \& Li, Y. Polymerized small-molecule acceptors for highperformance all-polymer solar cells. Angew. Chem. Int. Ed. 60, 4422-4433 (2021).

8. Wang, W. et al. Controlling molecular mass of low-band-gap polymer acceptors for high-performance all-polymer solar cells. Joule 4, 1070-1086 (2020).

9. Zhang, L. et al. $15.4 \%$ efficiency all-polymer solar cells. Sci. China Chem. 64, 408-412 (2021).

10. Fan, Q. et al. Over $14 \%$ efficiency all-polymer solar cells enabled by a low bandgap polymer acceptor with low energy loss and efficient charge separation. Energy Environ. Sci. 13, 5017-5027 (2020)

11. Choi, J., Kim, W., Kim, S., Kim, T.-S. \& Kim, B. J. Influence of acceptor type and polymer molecular weight on the mechanical properties of polymer solar cells. Chem. Mater. 31, 9057-9069 (2019).

12. Zhu, L. et al. Aggregation-induced multilength scaled morphology enabling $11.76 \%$ efficiency in all-polymer solar cells using printing fabrication. Adv. Mater. 31, 1902899 (2019).

13. Meng, Y. et al. $11.2 \%$ efficiency all-polymer solar cells with high open-circuit voltage. Sci. China Chem. 62, 845-850 (2019).

14. Yuan, J. et al. Single-junction organic solar cell with over $15 \%$ efficiency using fused-ring acceptor with electron-deficient core. Joule 3, 1140-1151 (2019).

15. Yuan, J. et al. Understanding energetic disorder in electron-deficient-corebased non-fullerene solar cells. Sci. China Chem. 63, 1159 (2020).

16. $\mathrm{Yu}, \mathrm{H}$. et al. Regio-regular polymer acceptors enabled by determined fluorination on end groups for all-polymer solar cells with $15.2 \%$ efficiency. Angew. Chem. Int. Ed. 60, 10137-10146 (2021).

17. Peng, F. et al. A universal fluorinated polymer acceptor enables all-polymer solar cells with $>15 \%$ efficiency. ACS Energy Lett. 5, 3702-3707 (2020).

18. $\mathrm{Fu}, \mathrm{H}$. et al. High efficiency (15.8\%) all-polymer solar cells enabled by a regioregular narrow bandgap polymer acceptor. J. Am. Chem. Soc. 143, 2665-2670 (2021)

19. Liu, L. et al. Graphdiyne derivative as multifunctional solid additive in binary organic solar cells with $17.3 \%$ efficiency and high reproductivity. Adv. Mater 32, 1907604 (2020)

20. $\mathrm{Wu}, \mathrm{Y}$. et al. Fine-tuning semiconducting polymer self-aggregation and crystallinity enables optimal morphology and high-performance printed allpolymer solar cells. J. Am. Chem. Soc. 142, 392-406 (2020).

21. $\mathrm{Na}, \mathrm{S}$.-I. et al. High performance roll-to-roll produced fullerene-free organic photovoltaic devices via temperature-controlled slot die coating. Adv. Funct Mater. 29, 1805825 (2019).

22. Qiu, B. et al. Highly efficient all-small-molecule organic solar cells with appropriate active layer morphology by side chain engineering of donor molecules and thermal annealing. Adv. Mater. 32, 1908373 (2020).

23. Jia, T. et al. $14.4 \%$ efficiency all-polymer solar cell with broad absorption and low energy loss enabled by a novel polymer acceptor. Nano Energy 72, 104718 (2020).

24. Du, J. et al. High-performance all-polymer solar cells: synthesis of polymer acceptor by a random ternary copolymerization strategy. Angew. Chem. Int Ed. 59, 15181-15185 (2020).

25. Yu, Z.-P. et al. Simple non-fused electron acceptors for efficient and stable organic solar cells. Nat. Commun. 10, 2152 (2019).

26. Jiang, $\mathrm{K}$. et al. Alkyl chain tuning of small molecule acceptors for efficient organic solar cells. Joule 3, 3020-3033 (2019).

27. Dong, S., Jia, T., Zhang, K., Jing, J. \& Huang, F. Single-component nonhalogen solvent-processed high-performance organic solar cell module with efficiency over 14\%. Joule 4, 2004-2016 (2020). 
28. Zhu, C. et al. Tuning the electron-deficient core of a non-fullerene acceptor to achieve over $17 \%$ efficiency in a single-junction organic solar cell. Energy Environ. Sci. 13, 2459-2466 (2020).

29. Lin, F. et al. Regio-specific selenium substitution in non-fullerene acceptors for efficient organic solar cells. Chem. Mater. 31, 6770-6778 (2019).

30. Wang, N. et al. Improving active layer morphology of all-polymer solar cells by dissolving the two polymers individually. Macromolecules 52, 2402-2410 (2019).

31. Fan, B. et al. Optimisation of processing solvent and molecular weight for the production of green-solvent-processed all-polymer solar cells with a power conversion efficiency over 9\%. Energy Environ. Sci. 10, 1243-1251 (2017).

32. $\mathrm{Hu}, \mathrm{H}$. et al. Design of donor polymers with strong temperature-dependent aggregation property for efficient organic photovoltaics. Acc. Chem. Res. 50, 2519-2528 (2017).

33. De Yoreo, J., N. A. J. M., S. Investigating materials formation with liquidphase and cryogenic TEM. Nat. Rev. Mater. 1, 16035 (2016).

34. Parry, A. L., Bomans, P. H., Holder, S. J., Sommerdijk, N. A. \& Biagini, S. C. Cryo electron tomography reveals confined complex morphologies of tripeptide-containing amphiphilic double-comb diblock copolymers. Angew. Chem. Int. Ed. 47, 8859-8862 (2008)

35. Jahng, J., Potma, E. O. \& Lee, E. S. Nanoscale spectroscopic origins of photoinduced tip-sample force in the midinfrared. PNAS 116, 26359-26366 (2019).

36. Ye, L. et al. Manipulating aggregation and molecular orientation in allpolymer photovoltaic cells. Adv. Mater. 27, 6046-6054 (2015).

37. Steyrleuthner, R. et al. Aggregation in a high-mobility n-type low-bandgap copolymer with implications on semicrystalline morphology. J. Am. Chem. Soc. 134, 18303-18317 (2012).

38. Panzer, F., Bässler, H. \& Köhler, A. Temperature induced order-disorder transition in solutions of conjugated polymers probed by optical spectroscopy. J. Phys. Chem. Lett. 8, 114-125 (2017).

39. Clark, J., Chang, J.-F., Spano, F. C., Friend, R. H. \& Silva, C. Determining exciton bandwidth and film microstructure in polythiophene films using linear absorption spectroscopy. Appl. Phys. Lett. 94, 163306 (2009).

40. Wirix, M. J. M., Bomans, P. H. H., Friedrich, H., Sommerdijk, N. A. J. M. \& de With, G. Three-dimensional structure of P3HT assemblies in organic solvents revealed by Cryo-TEM. Nano Lett. 14, 2033-2038 (2014).

41. Friedrich, H., Frederik, P. M., de With, G. \& Sommerdijk, N. A. Imaging of self-assembled structures: interpretation of TEM and cryo-TEM images. Angew. Chem. Int. Ed. 49, 7850-7858 (2010).

42. Nilsson, S., Bernasik, A., Budkowski, A. \& Moons, E. Morphology and phase segregation of spin-casted films of polyfluorene/PCBM blends. Macromolecules 40, 8291-8301 (2007).

43. Wang, T. \& Brédas, J.-L. Organic photovoltaics: understanding the preaggregation of polymer donors in solution and its morphological impact. $J$. Am. Chem. Soc. 143, 1822-1835 (2021).

44. Nowak, D. et al. Nanoscale chemical imaging by photoinduced force microscopy. Sci. Adv. 2, e1501571 (2016).

45. Wang, Z. et al. The coupling and competition of crystallization and phase separation, correlating thermodynamics and kinetics in OPV morphology and performances. Nat. Commun. 12, 332 (2021)

46. Jiang, K. et al. Pseudo-bilayer architecture enables high-performance organic solar cells with enhanced exciton diffusion length. Nat. Commun. 12, 468 (2021).

47. Jia, Z. et al. High performance tandem organic solar cells via a strongly infrared-absorbing narrow bandgap acceptor. Nat. Commun. 12, 178 (2021).

48. Hexemer, A. et al. A SAXS/WAXS/GISAXS beamline with multilayer monochromator. J. Phys. Conf. Ser. 247, 012007 (2010).

49. Rivnay, J., Mannsfeld, S. C. B., Miller, C. E., Salleo, A. \& Toney, M. F. Quantitative determination of organic semiconductor microstructure from the molecular to device scale. Chem. Rev. 112, 5488-5519 (2012).

50. Noriega, R. et al. A general relationship between disorder, aggregation and charge transport in conjugated polymers. Nat. Mater. 12, 1038-1044 (2013).

51. Hindeleh, A. M. \& Hosemann, R. Paracrystals representing the physical state of matter. J. Phys. C Solid State Phys. 21, 4155-4170 (1988).
52. Yao, J. et al. Cathode engineering with perylene-diimide interlayer enabling over $17 \%$ efficiency single-junction organic solar cells. Nat. Commun. 11, 2726 (2020).

53. $\mathrm{Ma}, \mathrm{Q}$. et al. Promoting charge separation resulting in ternary organic solar cells efficiency over 17.5\%. Nano Energy 78, 105272 (2020).

54. Proctor, C. M., Love, J. A. \& Nguyen, T.-Q. Mobility guidelines for high fill factor solution-processed small molecule solar cells. Adv. Mater. 26, 5957-5961 (2014).

55. Babics, M. et al. Solvent vapor annealing-mediated crystallization directs charge generation, recombination and extraction in BHJ solar Cells. Chem. Mater. 30, 789-798 (2018).

\section{Acknowledgements}

This work was supported by the National Key Research and Development Program of China (No. 2019YFA0705900) funded by MOST, NSFC (Nos. 51820105003, 21734008 and 61904181) and the Guangdong Major Project of Basic and Applied Basic Research (No. 2019B030302007). GIWAXS data were acquired at beamline 7.3.3 at the Advanced Light Source, which is supported by the Director, Office of Science, Office of Basic Energy Sciences, of the U.S. Department of Energy under Contract No. DE-AC02-05CH11231. Work by NCSU supported by ONR grant N000142012155.

\section{Author contributions}

J.D. synthesized and characterized the PSMAs of PS-Se and PN-Se. K.H., S.Q., and L.M. performed the device fabrication and characterization of the all-PSCs. J.Z. measured and analyzed transient absorption spectroscopy. X.K. participated in the synthesis of the PSMAs. J.Y. and B.G. performed the Cryo-TEM measurements. H.Y., I.A., and H.A. measured and analyzed the GIWAXS. Z.Z. participated discussion of the results. Y.L. and L.M. supervised the project, and J.D., J.Z., L.M., and Y.L. wrote the paper.

\section{Competing interests}

The authors declare no competing interests.

\section{Additional information}

Supplementary information The online version contains supplementary material available at https://doi.org/10.1038/s41467-021-25638-9.

Correspondence and requests for materials should be addressed to L.M., B.G., H.A. or Y.L.

Peer review information Nature Communications thanks Alex Jen, Ergang Wang and the other, anonymous, reviewer(s) for their contribution to the peer review of this work.

Reprints and permission information is available at http://www.nature.com/reprints

Publisher's note Springer Nature remains neutral with regard to jurisdictional claims in published maps and institutional affiliations.

Open Access This article is licensed under a Creative Commons Attribution 4.0 International License, which permits use, sharing, adaptation, distribution and reproduction in any medium or format, as long as you give appropriate credit to the original author(s) and the source, provide a link to the Creative Commons license, and indicate if changes were made. The images or other third party material in this article are included in the article's Creative Commons license, unless indicated otherwise in a credit line to the material. If material is not included in the article's Creative Commons license and your intended use is not permitted by statutory regulation or exceeds the permitted use, you will need to obtain permission directly from the copyright holder. To view a copy of this license, visit http://creativecommons.org/ licenses/by/4.0/

(C) The Author(s) 2021 